\title{
ESTUDO OBSERVACIONAL COMPARATIVO DE FRATURAS EM CRIANÇAS E ADOLESCENTES
}

\section{EPIDEMIOLOGIC COMPARATIVE STUDY OF FRACTURES IN CHILDREN AND ADOLESCENTS}

Roberto Guarniero', Rui Maciel de Godoy Junior², Eduardo Ambrosini Junior ${ }^{3}$, José Roberto Bevilacqua Guarniero³, Guilherme Bottino Martins ${ }^{3}$, Paulo José de Santana ${ }^{4}$, Marco Antonio Batista ${ }^{5}$, Carlos Eduardo Sanches Vaz ${ }^{5}$, Marcelo Yugi Cinagawa ${ }^{6}$

\section{RESUMO}

Objetivos: 1) Analisar comparativamente a incidência de fraturas na criança e no adolescente em três centros universitários de atendimento. 2) Estudar o acometimento segundo a faixa etária, o sexo, o tipo de acidente, a localização anatômica, a localização no osso, o tipo de fratura, a associação com outras lesões e o tratamento. 3) Em relação ao tipo de fratura e seu tratamento, avaliar se os programas de ensino e treinamento desenvolvidos atualmente, tanto na graduação como na pós-graduação Lato sensu (residência médica e especialização) estão condizentes com as necessidades do médico para o atendimento diário nos diferentes serviços de emergência de nosso País. Métodos: Estudo prospectivo transversal e observacional. Estudados os prontuários de 543 pacientes, no grupo etário de um dia de vida a 19 anos, atendidos em um período de três meses em três hospitais universitários, com uma fícha especialmente idealizada para determinar as características gerais das fraturas nesta série de pacientes. Resultados: Observamos a incidência de 531 fraturas (isoladas) e 12 luxações nos 543 pacientes, sendo, em relação ao sexo, 394 meninos $(72,5 \%)$ e 149 meninas $(27,4 \%)$. Segundo a etiologia dos traumatismos, o episódio "queda da própria altura" foi o que apresentou a maior incidência, seguido pela "queda de altura", caracterizando um traumatismo mais grave. Observamos o maior acometimento dos membros superiores em relação aos membros inferiores, sendo 404 fraturas nos superiores (com 11 luxações), representando 76,08\% das fraturas e 127 nos inferiores, que representam 23,91\%. Conclusões: 1) Em função da casuística estudada, podemos concluir que campanhas educativas devem ser realizadas com conteúdo que enfatize as causas e a prevenção da "queda da própria altura". 2) Os programas de treinamento do especialista devem enfatizar a maior ocorrência dos traumatismos e lesões dos membros superiores, bem como as suas causas.

Descritores - Fraturas Ósseas/epidemiologia; Traumatologia; Criança; Adolescente

\section{ABSTRACT}

Objectives: 1) To evaluate the incidence of fractures in children and adolescents in an universitarian hospital 2) to quantify the incidence based on age, sex gender, type of accident, anatomic location, association with other lesions, and treatment. 3) according to the type of fracture, to determine the adequacy of the treatment and training protocols, either for graduation or medical residency training in our medical services. Methods: A transversal prospective observational study. The medical records from 543 patients were analysed, aging one day to 19 years old, treated in a period of three months in our institutions, three different university hospitals, with a special protocol to determine the characteristics of the fractures in this series. Results: 531 fractures (isolated) were analysed along with 12 dislocations, 394 (72.5\%) male and 149 (27.4\%) female. The age ranged from one day to 19 years old. The most frequent etiology of those episodes was "fall from own height", followed by "fall from height" which refers to a more severe trauma. The upper limbs were most affected, with 404 fractures and eleven dislocations (six in the elbows, five in the shoulder) combining for $76.08 \%$ of all fractures against 127 fractures (combining for 23.91\% of all) in the lower limbs. In the casuistic is included two vertebral fractures (coccix) and one nasal fracture. Conclusions: The first obvious observation would concern the need of a directed prevention program, especially for the male teenagers, emphasizing the upper limbs. As of teaching programs and studies, it would be recommendable that the treatment of upper limbs trauma should be emphasized,in all of its segments.

Keywords - Bone Fractures/epidemiology; Traumatology; Child; Adolescent

1 - Professor Associado da Faculdade de Medicina da Universidade de São Paulo, Departamento de Ortopedia e Traumatologia - São Paulo, SP, Brasil.

2 - Professor Doutor da Faculdade de Medicina da Universidade de São Paulo, Departamento de Ortopedia e Traumatologia - São Paulo, SP, Brasil.

3 - Médico Ortopedista; Ex-Estagiário do Instituto de Ortopedia e Traumatologia do Hospital das Clínicas da FMUSP - São Paulo, SP, Brasil.

4 - Professor Adjunto da Faculdade Medicina da Universidade Estadual de Londrina - Londrina, PR, Brasil.

5 - Médico Assistente da Faculdade Medicina da Universidade Estadual de Londrina - Londrina, PR, Brasil.

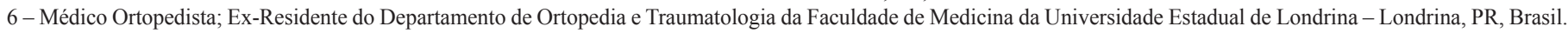

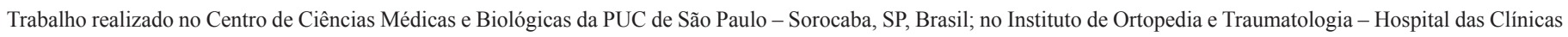
da FMUSP - São Paulo, SP, Brasil; e na Faculdade de Medicina da Universidade Estadual de Londrina - Londrina, PR, Brasil.

Correspondência: Rua João Moura, 627, 1ª andar, Pinheiros - 05412-011 - São Paulo, SP, Brasil. E-mails: robertoguarniero@gmail.com e editorab@gmail.com

Trabalho recebido para publicação: 24/08/2011, aceito para publicação: 31/08/2011.

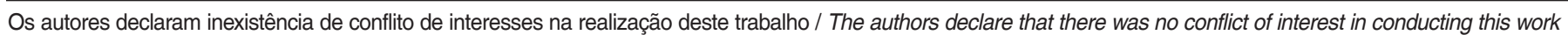




\section{INTRODUÇÃO}

Nos dias de hoje, os traumatismos permanecem como a principal causa de óbito nos adultos jovens em várias partes do mundo. Trabalhos epidemiológicos realizados nos Estados Unidos da América revelam a cifra estimada de 125.000 mortes por ano relacionadas a traumatismos ${ }^{(1-3)}$. Os acidentes e os traumatismos representam um dos maiores desafios para a saúde e o bem-estar das crianças e dos adolescentes. Alguns autores chamam a atenção para o fato de que as fraturas contribuem com uma variação de 10 a $25 \%$ entre todos os traumatismos ocorridos nessa faixa etária. Estes estudos são importantes para identificar e graduar a importância do problema, pois é também missão do médico não apenas tratar, mas auxiliar a evitar e prevenir os traumatismos. Como refere Dimeglio ${ }^{(4)}$, a Traumatologia Pediátrica é parte importante da Disciplina de Ortopedia Pediátrica, sendo que, no serviço do autor, o Hospital Lapeyronie, a cada ano, 1.000 casos de fraturas são tratados na urgência e três pacientes em cada 10 são hospitalizados.

Em relação à incidência de fraturas nas crianças e nos adolescentes, temos poucos $\operatorname{dados}^{(5,6)}$, pois são praticamente inexistentes os estudos epidemiológicos a este respeito em nosso meio. Portanto, este é um estudo relevante para o mapeamento desta situação. Deste modo, este trabalho tem como objetivos: 1) analisar e comparar a incidência de fraturas na criança e no adolescente em diferentes centros de atendimento; 2) comparar o acometimento segundo a faixa etária, o sexo, o tipo de acidente, a localização anatômica, a localização no osso, o tipo de fratura, a associação com outras lesões e o tratamento; e 3) em relação ao tipo de fratura e seu tratamento, avaliar se os programas de ensino desenvolvidos atualmente, tanto em nível de graduação como de pós-graduação Lato senso (residência médica e especialização), estão condizentes com as necessidades do médico para o atendimento diário nos diferentes serviços de emergência em nosso País.

Com o objetivo de tornar a amostragem mais abrangente e consistente e comparar os dados de diferentes regiões e padrões socioeconômicos, o levantamento de dados foi realizado nas regiões de Sorocaba (SP), São Paulo (Capital) e Londrina (PR), em três hospitais universitários.

Acreditamos que os resultados possam servir como referência para o desenvolvimento e o aprimoramento de programas educacionais e de treinamento em Trau- matologia Pediátrica, enfatizando os temas mais necessários ao aprendizado de alunos da graduação e da pós-graduação Lato senso. Também poderá auxiliar no direcionamento político de educação e de prevenção de fraturas no grupo etário pediátrico.

\section{MÉTODOS}

Foi realizado estudo prospectivo transversal e observacional. Foram analisados os prontuários de 543 pacientes no grupo etário de um dia de vida a 19 anos de idade, atendidos em um período de três meses nos diferentes serviços, com o objetivo de determinar as características das fraturas nesta série de pacientes. No Quadro 1, mostramos a ficha utilizada para a análise e o levantamento dos dados em nosso protocolo de estudo, com as seguintes variáveis: faixa etária, sexo, tipo de acidente, localização anatômica, localização no osso, tipo de fratura, associação com outras lesões e tratamento.

\section{RESULTADOS}

Verificamos a incidência de 531 fraturas (isoladas) e 12 luxações nos 543 pacientes do estudo, sendo, em relação ao sexo, 394 meninos e 149 meninas (Tabelas 1 e 2). A idade dos pacientes variou de um dia de vida, como mínimo, ao máximo de 19 anos. Na Tabela 3, mostramos a distribuição das fraturas segundo diferentes faixas etárias. Na Tabela 4, mostramos a distribuição segundo a etiologia dos traumatismos. O episódio "queda da própria altura" foi o que apresentou a maior incidência, seguido pela "queda de altura", caracterizando um traumatismo mais grave. No Quadro 2, correlacionamos a gravidade da etiologia com o tipo de fratura (localização) e o seu tratamento.

Observamos o maior acometimento dos membros superiores em relação aos membros inferiores, sendo 404 fraturas nos superiores (com 11 luxações), representando 76,08\% das fraturas, e 127 nos inferiores, que representam 23,91\%. As 11 luxações nos membros superiores foram cinco do ombro e seis do cotovelo; uma luxação ocorreu nos membros inferiores, na articulação metatarsofalângica. No material estudado estão incluídas duas fraturas na região da coluna vertebral (cóccix) e uma fratura dos ossos próprios do nariz (nasal).

Nas Tabelas 5, 6 e 7, mostramos a incidência em relação ao osso atingido e segundo a localização no membro superior ou no inferior. 
Quadro 1 - Ficha de avaliação para levantamento dos dados.

\begin{tabular}{|c|c|c|c|c|c|c|c|}
\hline \multicolumn{8}{|l|}{ Número: } \\
\hline D. nasc.: & & & D. acid.: & & & & \\
\hline \multirow[t]{4}{*}{ Idade: } & & Sexo: & Masculino ( ) & Feminino ( ) & & & \\
\hline & \multirow{3}{*}{\multicolumn{2}{|c|}{ Tipo de traumatismo: }} & $\begin{array}{c}\text { Queda alt. } \\
(\quad)\end{array}$ & $\begin{array}{l}\text { Queda } \\
(\quad)\end{array}$ & 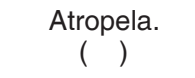 & $\begin{array}{c}\text { Bicicleta } \\
(\quad)\end{array}$ & $\begin{array}{c}\text { Esporte } \\
\left(\begin{array}{l}\text { ( })\end{array}\right.\end{array}$ \\
\hline & & & Moto & Auto & Skate & Patim & \\
\hline & & & ( ) & ( ) & $(\quad)$ & ( ) & \\
\hline \multicolumn{8}{|c|}{ Outro: } \\
\hline Fratura: & & Exposta & () & Fechada & () & & \\
\hline \multirow[t]{2}{*}{ MMSS } & Clavícula ( ) & Úmero ( ） & Cotovelo ( ) & Antebraço ( ) & Punho ( ) & \multirow{2}{*}{\multicolumn{2}{|c|}{ Osso carpo ( ) }} \\
\hline & Metacarpo ( ) & Falange ( ) & Polegar ( ) & & & & \\
\hline \multirow[t]{3}{*}{ MMII } & Bacia ( ) & & Fêmur prox. ( ) & Fêmur ( ) & Joelho ( ) & & \\
\hline & Tíbia ( ) & Fíbula ( ) & Tornozelo ( ) & Osso tarso ( ) & & & \\
\hline & Metatarso ( ) & Falange ( ) & Hálux ( ) & & & & \\
\hline \multirow[t]{2}{*}{ Coluna } & Cervical ( ) & Dorsal ( ) & Lombar ( ) & & & & \\
\hline & \multicolumn{2}{|c|}{ Localização no osso: } & Diáfise ( ) & Metáfise ( ) & Epífise ( ) & & \\
\hline Desvio: & Com ( ) & Sem ( ) & & & & & \\
\hline \multirow[t]{2}{*}{ Fratura: } & Simples ( ) & Cominut. ( ) & Espiralada ( ) & Oblíqua ( ) & Transver. ( ) & Outra: ( ) & ) \\
\hline & Deslocam & to epifisário/tipo: & & I ( ) & II ( ) & III ( ) & $\operatorname{IV}(\quad)$ \\
\hline Luxação: & $\operatorname{Sim}(\quad)$ & Não（） & Qual: & & & & \\
\hline
\end{tabular}

Tabela 1 - Tipo de lesão e frequência.

\begin{tabular}{c|c}
\hline Lesão & Frequência (\%) \\
\hline Fraturas & $531(97,79)$ \\
\hline Luxações & $12(2,20)$ \\
\hline Total & $543(100)$ \\
\hline
\end{tabular}

Tabela 2 - Distribuição segundo o sexo.

\begin{tabular}{c|c}
\hline Sexo & Frequência (\%) \\
\hline Masculino & $394(72,5)$ \\
\hline Feminino & $149(27,4)$ \\
\hline Total & $543(100)$ \\
\hline
\end{tabular}

Tabela 3 - Distribuição das fraturas segundo diferentes faixas etárias.

\begin{tabular}{c|c|c|c|c}
\hline Faixa etária & SP & Sorocaba & Londrina & Total \\
\hline $0-3$ anos & $8(14,81 \%)$ & $15(29,76 \%)$ & $32(11,68 \%)$ & $55(10,12 \%)$ \\
\hline $4-7$ anos & $10(18,51 \%)$ & $42(19,53 \%)$ & $81(29,56 \%)$ & $133(24,49 \%)$ \\
\hline $8-11$ anos & $15(27,77 \%)$ & $64(29,76 \%)$ & $87(31,75 \%)$ & $166(30,57 \%)$ \\
\hline 12 ou mais anos & $21(38,88 \%)$ & $94(43,72 \%)$ & $74(27,01 \%)$ & $189(34,80 \%)$ \\
\hline Total & 54 & 215 & 274 & 543 \\
\hline
\end{tabular}


Tabela 4 - Etiologia dos traumatismos em 543 pacientes.

\begin{tabular}{l|c|c|c}
\hline \multicolumn{1}{c|}{ Causa } & SP (\%) & Sorocaba (\%) & Londrina (\%) \\
\hline Queda - própria altura & $30(55,55)$ & $152(70,69)$ & $105(38,32)$ \\
\hline Queda de altura & 0 & $20(9,30)$ & $60(22,72)$ \\
\hline Esporte & $1(1,85)$ & $17(7,90)$ & $10(3,64)$ \\
\hline Bicicleta & $5(9,25)$ & $9(4,18)$ & $36(13,14)$ \\
\hline Traumatismo direto & $13(24,07)$ & $5(2,32)$ & $29(10,57)$ \\
\hline $\begin{array}{l}\text { Acidente de automóvel - } \\
\text { passageiro }\end{array}$ & 0 & $2(0,93)$ & $3(1,09)$ \\
\hline Acidente com skate & $2(0,93)$ & 0 & 0 \\
\hline Entorse tornozelo e pé & $1(1,85)$ & $2(0,93)$ & $3(1,09)$ \\
\hline Atropelamento - pedestre & $3(5,55)$ & $1(0,46)$ & $7(2,55)$ \\
\hline $\begin{array}{l}\text { Acidente com motocicleta } \\
- \text { passageiro }\end{array}$ & 0 & $1(0,46)$ & $5(1,83)$ \\
\hline Queda de cavalo & 0 & $1(0,46)$ & $4(1,46)$ \\
\hline $\begin{array}{l}\text { Ferimento por arma de } \\
\text { fogo }\end{array}$ & 0 & $1(0,46)$ & $1(0,36)$ \\
\hline Acidente com patins & 0 & $1(0,46)$ & $3(1,09)$ \\
\hline $\begin{array}{l}\text { Vitima de maus tratos } \\
\text { infantis }\end{array}$ & 0 & $1(0,46)$ & 0 \\
\hline Traumatismo parto & $1(1,85)$ & 0 & $1(0,36)$ \\
\hline Fogos de artifício & 0 & 0 & $2(0,72)$ \\
\hline Cortador de grama & 0 & 0 & $1(0,36)$ \\
\hline & $54(100)$ & $215(100)$ & $274(100)$ \\
\hline
\end{tabular}

Tabela 7 - Distribuição da localização das fraturas segundo o osso acometido. Membro inferior.

\begin{tabular}{c|c|c|c|c}
\hline MMII (osso) & SP (\%) & Sorocaba (\%) & Londrina (\%) & Total \\
\hline Fêmur & 0 & $8(14,28)$ & $9(14,75)$ & $17(13,38)$ \\
\hline $\begin{array}{c}\text { Supracondiliana } \\
\text { do fêmur }\end{array}$ & $1(10)$ & $1(1,78)$ & 0 & $2(1,57)$ \\
\hline Patela & 0 & $1(1,78)$ & $1(1,63)$ & $2(1,57)$ \\
\hline Tíbia e fíbula & $1(10)$ & $15(26,78)$ & $4(6,55)$ & $20(15,74)$ \\
\hline Fíbula & $1(10)$ & $2(3,57)$ & $1(1,63)$ & $4(3,14)$ \\
\hline Tíbia & 0 & 0 & $21(34,42)$ & $21(16,53)$ \\
\hline Tornozelo & $2(20)$ & $1(1,78)$ & $4(6,55)$ & $7(5,51)$ \\
\hline Tálus & 0 & $1(1,78)$ & $2(3,27)$ & $3(2,36)$ \\
\hline Calcâneo & 0 & $4(7,14)$ & $1(1,63)$ & $5(3,93)$ \\
\hline Metatarsal & $2(20)$ & $13(23,21)$ & $5(8,19)$ & $20(15,7)$ \\
\hline Hálux & $2(20)$ & $4(7,14)$ & $1(1,63)$ & $7(5,51)$ \\
\hline $\begin{array}{c}\text { Falange } \\
\text { pododáctilos }\end{array}$ & $1(10)$ & $6(10,71)$ & $12(19,67)$ & $19(14,96)$ \\
\hline Total & $10(100)$ & $56(100)$ & $61(100)$ & $127(100)$ \\
\hline
\end{tabular}

Tabela 5 - Distribuição da localização das fraturas segundo o membro acometido.

\begin{tabular}{c|c|c|c|c}
\hline Localização & SP (\%) & Sorocaba (\%) & Londrina (\%) & Total (\%) \\
\hline MMSS & $40(80)$ & $156(73,58)$ & $208(77,32)$ & $404(76,08)$ \\
\hline MMII & $10(20)$ & $56(26,41)$ & $61(22,67)$ & $127(23,91)$ \\
\hline TOTAL & $50(100)$ & $212(100)$ & $269(100)$ & $531(100)$ \\
\hline
\end{tabular}

Tabela 6 - Distribuição da localização das fraturas segundo o osso acometido. Membro superior.

\begin{tabular}{|c|c|c|c|c|}
\hline MMSS (osso) & SP (\%) & Sorocaba (\%) & Londrina (\%) & Total (\%) \\
\hline Omoplata & 0 & $1(0,64)$ & $3(1,44)$ & $4(0,99)$ \\
\hline Clavícula & $6(15)$ & $12(7,69)$ & $28(13,46)$ & $46(11,38)$ \\
\hline Úmero & $2(5)$ & $14(8,97)$ & $5(2,40)$ & $21(5,19)$ \\
\hline Supracondiliana úmero & $4(10)$ & $10(6,41)$ & $14(6,73)$ & $28(6,93)$ \\
\hline Capítulo umeral & $1(2,5)$ & 0 & $4(1,92)$ & $5(1,23)$ \\
\hline Côndilo umeral & 0 & 0 & $1(0,48)$ & $1(0,24)$ \\
\hline Olecrano & 0 & 0 & $1(0,48)$ & $1(0,24)$ \\
\hline Antebraço (2) & $3(7,5)$ & $28(17,94)$ & $80(38,46)$ & $111(27,47)$ \\
\hline Rádio & $2(5)$ & $34(21,79)$ & 0 & $36(8,9)$ \\
\hline Ulna & $1(2,5)$ & 0 & 0 & $1(0,24)$ \\
\hline Punho & $7(17,5)$ & $28(17,94)$ & $49(23,55)$ & $84(20,79)$ \\
\hline Carpo & 0 & $1(0,64)$ & 0 & $1(0,24)$ \\
\hline Escafoide & $2(5)$ & $1(0,64)$ & $1(0,48)$ & $4(0,99)$ \\
\hline Metacarpiano & $1(2,5)$ & $10(6,41)$ & $5(2,40)$ & $16(3,96)$ \\
\hline Falange polegar & $2(5)$ & $2(1,28)$ & 0 & $4(0,99)$ \\
\hline Falange dedos & $9(22,5)$ & $15(9,61)$ & $17(8,17)$ & $41(10,14)$ \\
\hline Total & $40(100)$ & $156(100)$ & $208(100)$ & $404(100)$ \\
\hline
\end{tabular}


Quadro 2 - Correlação entre a etiologia e o tipo de fratura e seu tratamento.

\begin{tabular}{|c|c|c|}
\hline Etiologia & Tipo de fratura-localização & Tratamento utilizado \\
\hline Bicicleta & Antebraço & Imobilização \\
\hline Bicicleta & Antebraço & Imobilização \\
\hline Bicicleta & Nariz & Redução \\
\hline Bicicleta & Falange dedo da mão & Imobilização \\
\hline Bicicleta & Tíbia + Fíbula & Redução +Imobilização \\
\hline Bicicleta & Rádio & Redução + Imobilização \\
\hline Bicicleta & Rádio & Imobilização \\
\hline Bicicleta & Clavícula & Imobilização \\
\hline Bicicleta & Escafoide - mão & Imobilização \\
\hline Motocicleta & Metacarpo & Imobilização \\
\hline Queda de cavalo & Antebraço & Imobilização \\
\hline Acidente automobilístico & Fêmur & Cirurgia/Osteossíntese \\
\hline Acidente automobilístico & Clavícula & Imobilização \\
\hline Ferimento por arma de fogo & Antebraço & Cirurgia/Osteossíntese \\
\hline Acidente com patins & Rádio & Redução + Imobilização \\
\hline Acidente com skate & Antebraço & Redução + Imobilização \\
\hline Acidente com skate & Úmero & Imobilização \\
\hline Atropelamento & Fêmur & Redução + Aparelho gessado \\
\hline
\end{tabular}

\section{DISCUSSÃO}

O objetivo principal deste trabalho foi fazer uma análise descritiva das características das fraturas encontradas em uma série de pacientes pediátricos e adolescentes (até 18 anos de idade). Concordamos com Dimeglio ${ }^{(4)}$ quando o autor afirma que a traumatologia pediátrica, pela frequência de lesões, é um problema de saúde pública. Entretanto, antes de elaborar e iniciar programas de prevenção dos acidentes e de fraturas dirigidos à criança e ao adolescente, necessitamos de estudos epidemiológicos deste tipo para a seleção das ações preventivas.

A análise dos resultados do nosso estudo mostra características interessantes e importantes em relação às fraturas encontradas no grupo avaliado. Evidentemente, a primeira delas é o acometimento dos meninos em relação às meninas, como vemos nas Tabelas 1 e 2 . Neste trabalho, esta proporção foi de, aproximadamente, três meninos para cada menina $(72,5 \%$ para $27,4 \%)$, o que, de certa forma, está de acordo com a bibliografia consultada $^{(1,4,5-10)}$.

$\mathrm{Na}$ avaliação dos nossos resultados, em relação à idade dos pacientes, verificamos que aproximadamente $65 \%$ das fraturas têm prevalência entre as idades de um e 11 anos, com os restantes 35\% ocorrendo nos pacientes com 12 anos e maiores. Estes valores estão de acordo com a literatura, pois, como refere Dimeglio(4), encontramos até $55 \%$ das fraturas na faixa etária de um a 11 anos. Este autor cita que até os seis anos de idade se agrupam $18 \%$ de todas as fraturas, sendo que entre os seis e os 11 anos estariam presentes em torno de $40 \%$ delas; chama a atenção para o fato de que cerca de $80 \%$ das fraturas ocorrem após os seis anos de idade. Também concordamos em parte com estes dados mencionados por Dimeglio ${ }^{(4)}$, pois em nosso estudo cerca de $65 \%$ das fraturas ocorreram nos pacientes maiores de oito anos. Entretanto, quando analisamos os resultados por faixas etárias diferenciadas (zero a três anos, quatro a sete anos, oito a 11 anos e 12 anos e maiores) (Tabela 3), verificamos no nosso material a maior incidência global nos adolescentes ( $35 \%$ em média) nos pacientes com 12 anos de idade e maiores, o que também concorda com outros recentes levantamentos efetuados no nosso meio, como mostra o trabalho de Carvalho Júnior et $a l^{(6)}$.

O episódio "queda da própria altura" foi o que apresentou a maior incidência, seguido pela "queda de altura", caracterizando um traumatismo mais grave como se observa na Tabela 4.

Uma das inferências, advinda do presente levantamento de dados, é em relação aos possíveis programas de prevenção. A necessidade primordial é o direcionamento principal de campanhas educativas para os adolescentes do sexo masculino. O segundo ponto impor- 
tante está relacionado com a localização das fraturas, devido à predominância do acometimento do membro superior (Tabela 5). Temos praticamente mais de 50\% das fraturas localizadas nos ossos do antebraço, isoladamente ou em ambos, e no punho. Felizmente, a grande maioria destas lesões são fraturas simples, cujo tratamento necessário consiste na imobilização da região atingida, sem apresentar grandes complicações.

Em relação aos programas de ensino para os alunos da graduação e, também, em alguns programas de especialização, classificaríamos como pontos importantes:

\section{Programa Ideal Básico, fundamentado em:}

a) Princípios do tratamento conservador das fraturas, redução incruenta e técnicas de aplicação dos aparelhos gessados;

b) Princípios gerais de fixação externa e sua aplicabilidade no tratamento das fraturas de crianças e adolescentes;

c) Princípios gerais de osteossíntese e sua aplicabilidade no tratamento das fraturas de crianças e adolescentes; d) Opções de tratamento de acordo com a idade do paciente, a classificação da fratura e o desvio dos fragmentos;

e) Fraturas e luxações do cotovelo; e

f) Fraturas do antebraço, punho e mão.

2. Programa de Prevenção, com 10 pontos básicos:

a) Informar as famílias;

\section{REFERÊNCIAS}

1. Armstrong PF, Joughin VE, Clarke HM. Pediatric fractures of the forearm wrist and hand. In: Green NE, Swiotkowski MF. Skeletal trauma in children. Philadelphia: Saunders; 1994. p.127.

2. Reed HM. Fractures and dislocations of the extremities in chidren. J Trauma 1977;17(5):351-4.

3. Wilkins $\mathrm{K}$. The incidence of fractures in children. In: Rockwood CA, Wilkins K Beaty JH. Fractures in children. Philadelphia: Lippincott-Raven; 1996. p. 3.

4. Dimeglio A. Epidemiologia de las fracturas en el niño. In: DePablos J, Gonzalez-Herranz, P. Apuntes de fracturas infantiles. Madri: Ediciones Ergon; 1999. p.11.

5. Borges JLP, Laghi R, Pitagoras T, Calieron LG. Fraturas isoladas do terço Dista do rádio na criança. Rev Bras Ortop. 2000;35(1):88-93. b) Informar as associações esportivas;

c) Adequar as residências;

d) Avisar as escolas e adequar os locais de recreação e as redondezas das escolas e colégios;

e) Incrementar e valorizar os programas de prevenção de acidentes de trânsito;

f) Mobilizar as associações de proteção de consumidores;

g) Mobilizar e informar a mídia;

h) Lidar com toda a cadeia de assistência à saúde;

i) Realizar avaliações de despesas com os tratamentos para sensibilizar as autoridades políticas; e

j) Valorizar os efeitos causados às crianças.

\section{CONCLUSÕES}

1) $\mathrm{O}$ estudo demonstra a necessidade da realização de campanhas, cujo conteúdo enfatize as causas e a prevenção da "queda da própria altura" nas crianças.

2) Os programas de treinamento do especialista devem abranger e estudar a maior frequência dos traumatismos dos membros superiores, incluindo as suas causas, bem como seu tratamento conservador e/ou cirúrgico.

\section{AGRADECIMENTO}

Os autores agradecem o auxílio na coleta dos dados dos Drs. Gianmarco Líbano, José Francisco V. Ayres, Ayres, Rossana A. Assanuma e Fernando Takao Cinagawa.
6. Carvalho Júnior LH, Cunha FM, Ferreira FS, Morato AEP, Rocha LHA., Medeiros RF. Lesões ortopédicas traumáticas em crianças e adolescentes. Rev Bras Ortop. 2000;35(1):80-7.

7. Aktas S, Saridogan K, Morala U, Ture M. Patterns of single segment nonphyseal extremity fractures in children. Int Orthop.1999;23(6):345-7.

8. Cheng JC, Shen WY. Limb fracture pattern in different pediatric age groups: a study of 3.350 children. J Orthop Trauma. 1993;7(1):15-22.

9. Landin LA. Epidemiology of children's fractures. J Pediatr Orthop B. 1997;6(2):79-83.

10. Worlock P, Stower M. Fracture pstterns in Nottingham children. J Pediatr Orthop 1986;6(6):656-60. 